\title{
Studies on the synthesis and fluorescent properties of long-chained 2-(5-alkyl-1, 3, 4-oxadiazol-2-yl)-3H-benzo[f]chromen-3-ones
}

\author{
Rajesha ${ }^{\text {a }}$ and H. S. Bhojya Naik, ${ }^{\text {a }}$ H. N. Harish Kumar, ${ }^{\text {K }}$ K. M. Hosamani, ${ }^{\text {c }}$ \\ and K. M. Mahadevan ${ }^{b^{*}}$ \\ ${ }^{a}$ Department of Post Graduate Studies and Research in Industrial Chemistry, Kuvempu \\ University, Shankaraghatta, Karnataka 577 451, India \\ ${ }^{b}$ Department of Post Graduate Studies and Research in Chemistry, Kuvempu University, \\ Shankaraghatta, Karnataka 577 451, India \\ ${ }^{c}$ Department of Studies in Chemistry, Karnataka University, Dharwad, \\ Karnataka, India \\ E-mail: madykmm@yahoo.co.uk
}

\begin{abstract}
The synthesis and fluorescent properties of some new benzocoumaryl heterocyclic molecules are presented. Condensation of 2-hydroxy-1-naphthaldehyde with diethyl malonate in the presence of catalytic amount of piperidine in ethanol affords benzocoumarin-3-ethyl carboxylate 2 in fairly good yield (90\%). Further the compound $\mathbf{2}$ on treatment with hydrazine hydrate afforded compound 3 with excellent yield (95\%). Thus compounds 4 a-e have been obtained by direct cyclization of 3 with various fatty acids in the presence of $\mathrm{POCl}_{3}$. The structures of all newly synthesized compounds were confirmed by IR, ${ }^{1} \mathrm{H}$ NMR, ${ }^{13} \mathrm{C}$ NMR and UPLC-Mass spectral data. Fluorescent experimental results revealed remarkable photoluminescence properties. Luminescent properties of all newly synthesized compounds were determined and observed that they exhibited strong blue-green fluorescent properties. The fluorescence spectral properties of compounds 4a-e are similar to each other and the Stoke's shift ranges from 54 to $59 \mathrm{~nm}$. Both the absorption and fluorescence maxima of the benzocoumarin-oxadiazole compounds showed good bathochromic shift.
\end{abstract}

Keywords: Fluorescence, benzocoumarins, benzocoumarin-3-ethylcarboxylate, benzocoumarin oxadiazolyls, phosphorous oxychloride, fatty acid oxadiazolyls

\section{Introduction}

Fluorescent heterocyclic compounds are of immense interest as functional materials in the emitters of electroluminescence devices and in the molecular probes used for biochemical 
research, as well as in the traditional textile and polymer fields. ${ }^{1-3}$ In particular, fluorescent dye materials whose fluorescence emission occur at a longer wavelength in the red light region play a leading role in full color electroluminescence displays. Heterocyclic fluorophores are useful materials in the search for new biologically active compounds and diagnostic methods. ${ }^{4}$ Fluorescent chromophores are generally known to have planar and rigid pi-conjugated systems, and many fluorescent chromophores are based on rigid ring systems such as stilbene, coumarin, naphthalimide, perylene and rodamine. Our research group is interested in the chemistry of oxygen containing heterocyclic molecules. Coumarin is a naturally occurring constituent of many plants and essential oils, including tonka beans, sweet clover, woodruff, oil of cassia, and lavender. It derives its name from the plant Coumarouna odorata. Vogel isolated and purified coumarin from the tonka bean (Dipreryx odorata) in $1822^{5}$. Coumarin derivatives exhibited useful and diverse activity in pharmaceuticals, fragrances, agrochemicals, insecticides and polymer science have become the most extensively investigated and commercially significant group of organic fluorescent materials in recent years. ${ }^{6-9}$ Coumarins played vital role in electro photographic and electroluminescent devices and laser dyes. Several 3-substituted 7hydroxycoumarins rank among the most efficient photostable laser dyes emitting in blue green region of the visible spectrum. The lasing range covered by coumarin dyes is appreciably extended when the 3 -substituent is a heterocyclic moiety. ${ }^{10,11}$ Therefore it is relevant to design and synthesize coumarins bearing different heterocycles at the 3-position with the aim to obtain a new photostable laser dyes having rigid structures that are tunable over a wide wavelength range within the visible spectrum. In recent years, the use of coumarins as fluorescent labels for a variety of compounds has been reported. ${ }^{12,13}$ Their benzo counterparts, namely benzocoumarins, have been less studied. Akira Takadate et al., studied fluorescence properties of coumarins and benzocoumarins. ${ }^{14}$ They observed that benzocoumarins and their derivatives with different heterocycles at position-3 are strongly fluorescent. Solubility is the main hurdle for the heterocyclic compounds to screen for various activities, long chain aliphatic carbon system enhance the solubility of the oxadiazoles when compared to aromatic ring systems. Hence we thought of constructing benzocoumarin oxadiazole ring using various fatty acids. Moreover these fatty acids are easily available. The synthesized compounds showed high fluorescent properties.

\section{Results and Discussion}

Benzocoumarins coupled with oxadiazolyl ring system are in general comparatively easy to prepare and numerous derivatives have been designed and prepared for potential use as biologically and fluorescent active materials. The classical synthesis of benzocoumarins involves condensation of aromatic hydroxy aldehyde with an active methylene compound. The reaction is facile and is most widely used synthetic method for both benzocoumarins and its derivatives. The various benzocoumaryl oxadiazoles 4a-e have been synthesized by benzocoumaryl-3ethylcarbohydrazide $\mathbf{3}$. The benzocoumarin-3-carbohydrazide $\mathbf{3}$ was prepared in an excellent 
yield from benzocoumarin-3-ethylecarboxylate 2 on treatment with hydrazine hydrate. The benzocoumarin-3-ethylcarboxylate was in return obtained by Knoevenagel condensation between 2-hydroxy-1-naphthaldehyde and diethylmalonate in the presence of catalytic amount of piperidine.

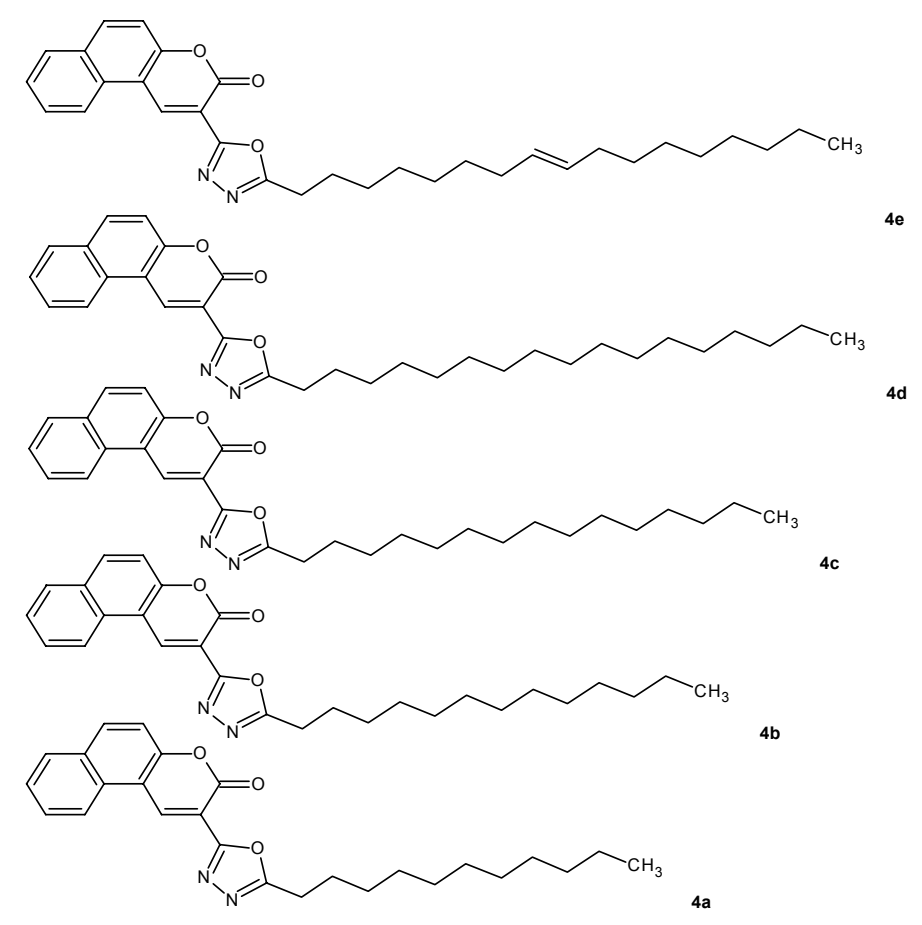

Figure 1. The structure of 4a-e.

The structure of compound 3 was established by ${ }^{1} \mathrm{H}$ NMR and mass spectral data. ${ }^{1} \mathrm{H}$ NMR of compound 3 revealed presence of singlet at $\delta 9.9 \mathrm{ppm}$ for one proton of $\mathrm{NH}\left(\mathrm{D}_{2} \mathrm{O}\right.$ exchangeable) group and another singlet at $\delta 4.7 \mathrm{ppm}$ for two protons of $\mathrm{NH}_{2}\left(\mathrm{D}_{2} \mathrm{O}\right.$ exchangeable), The formation of the compound $\mathbf{3}$ is also supported by its mass spectrum. The molecular ion peak was observed at MS (M+1) 255 (100\%) for compound 3, that confirms conversion of benzocoumarin carboxylate to benzocoumarin carbohydrazide 3 . Further the compound $\mathbf{3}$ has been exploited to construct an oxadiazole ring linked to benzocoumarin at position-3. The compound $\mathbf{3}$, on refluxing with various fatty acids in the presence of $\mathrm{POCl}_{3}$, affords the benzocoumarin-3-oxadiazoles 4 a-e. ${ }^{15}$ 
<smiles>CCOC(=O)COC(=O)OCC</smiles><smiles>CCOC(=O)c1cc2c(ccc3ccccc32)oc1=O</smiles><smiles>[R]Oc1nnc(-c2cc3c(ccc4ccccc43)oc2=O)o1</smiles>

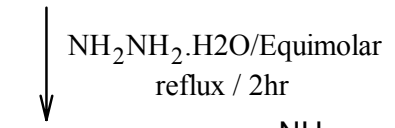<smiles>NNC(=O)c1cc2c(ccc3ccccc32)oc1=O</smiles>

3

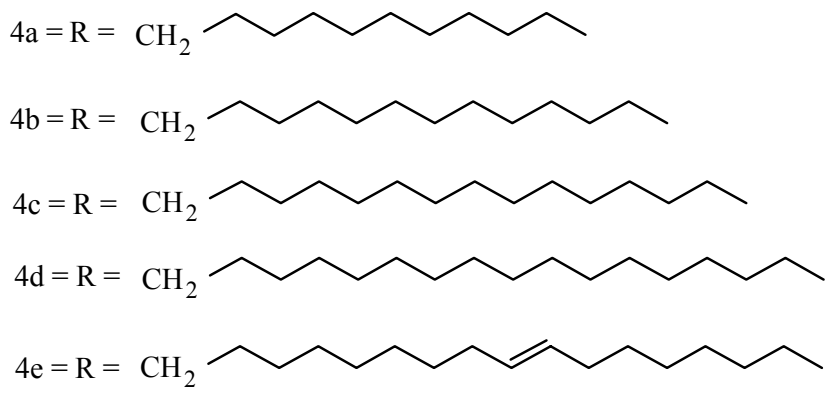

\section{Scheme 1}

General synthetic procedure for ethyl benzocoumarin-3-carboxylate 2, benzocoumarin-3carbohydrazide 3 and 3-benzocoumarin-5-(alkyl)-1, 3, 4 oxadiazoles 4a-e.

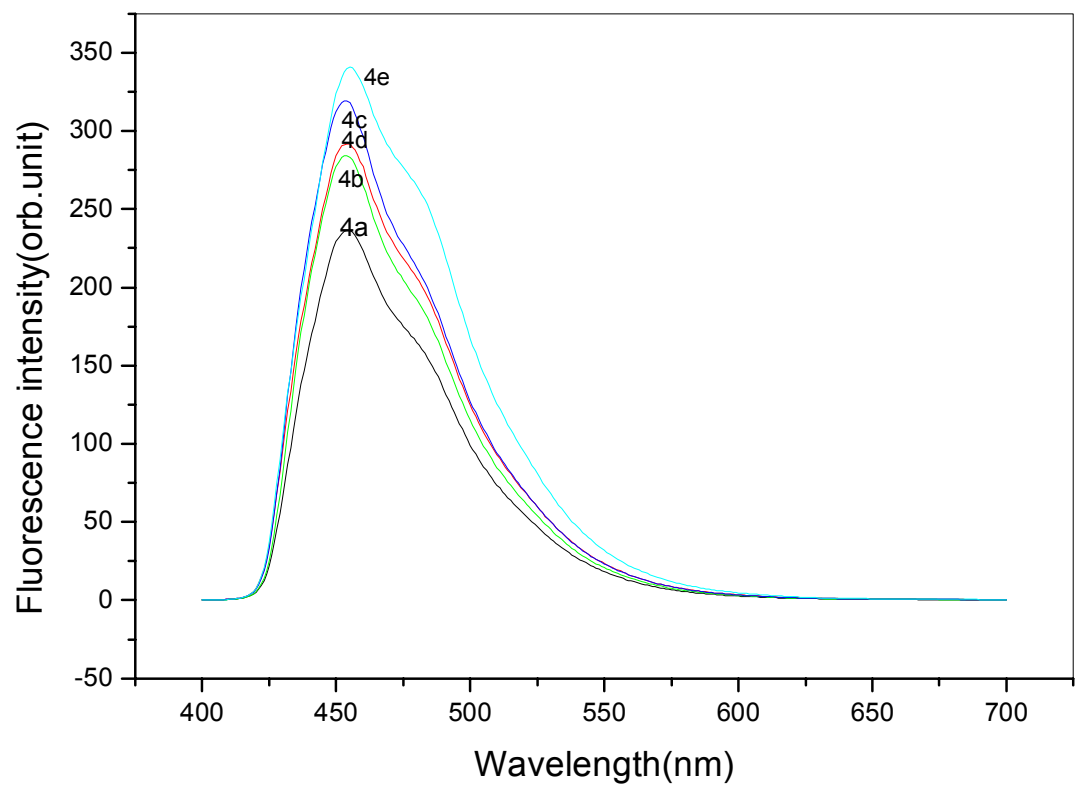

Figure 2. The fluorescence spectra of benzocoumarin oxadiazoles 4a-e in chloroform. 
The formation of 4a-e was verified with ${ }^{1} \mathrm{H}$ NMR and mass spectroscopic analysis. For example ${ }^{1} \mathrm{H}$ NMR spectra of $\mathbf{4 b}$ indicates that triplet at $\delta 3.0 \mathrm{ppm}$, corresponds to two protons confirms the $\mathrm{CH}_{2}$ group attached to oxadiazole ring. Triplet at $\delta 0.9 \mathrm{ppm}$ corresponds to three protons revealing the presence of $\mathrm{CH}_{3}$ group of terminal alkyl side chain and peaks at $\delta 1.9$ to 1.2 confirms remaining $\mathrm{CH}_{2}$ protons (22 protons). The peaks at $\delta 7.2$ to $8.4 \mathrm{ppm}$ correspond to six protons and corresponding to aromatic protons of benzocoumarin ring and singlet at $\delta 9.4 \mathrm{ppm}$ for one proton reveals the presence of $\mathrm{CH}$ proton of lactone ring in benzocoumarin nucleus. The molecular ion peaks exhibited at MS (M+1) 419 (100\%), 447.5 (100\%), 475.6 (100\%), 503 (100\%), and 501.13 (100\%) for compounds 4a-e respectively, which is crucial for fluorescence. All the newly synthesized compounds have been characterized by elemental analysis and spectroscopic data. The spectral details of all these are given in experimental section.

Table 1. Fluorescence spectral data of compounds 4a-e in chloroform

\begin{tabular}{ccccc}
\hline Compound & \multicolumn{2}{c}{$\begin{array}{c}\text { Maximum } \\
\text { wavelength }(\mathrm{nm})\end{array}$} & Stoke's shift (nm) & $\Delta v / \mathrm{cm}^{-1}$ \\
\cline { 2 - 3 } & Excitation & Emission & & \\
\hline 4a & 396 & 455 & 59 & 3300 \\
4b & 398 & 454 & 56 & 3100 \\
4c & 396 & 453 & 57 & 3200 \\
4d & 398 & 454 & 56 & 3100 \\
4e & 402 & 456 & 54 & 3000
\end{tabular}

\section{UV-visible and fluorescence spectral data analysis}

The experimental UV-visible spectra of benzocoumarin oxadiazolyls 4a-e in chloroform were obtained and are shown in Table 1. The emission spectra of compounds 4a-e are reproduced in Figure 2. In the visible region the absorption bands of all the compounds experience a good bathochromic shift. The fluorescence spectral properties (Table 1) of compounds 4a-e are similar to each other and the Stoke's shift ranges from 54 to $59 \mathrm{~nm}$. According to the literature, several 3-substituted-7-hydroxycoumarins rank among the most efficient photostable laser dyes emitting in the blue green region of the visible spectrum. ${ }^{10,11}$ The newly synthesized compounds 4 a-e also emit in the blue green region of the visible spectrum that is better than 3-substituted-7-hydroxy coumarins.

\section{Conclusions}

A simple, efficient and general method has been developed for the synthesis of oxadiazalyl benzocoumarin derivatives through one pot reaction of aliphatic carboxylic acids and benzocoumarin-3-carboxyhydrazide in the presence of $\mathrm{POCl}_{3}$ at reflux condition. All these 
compounds are hitherto unknown in literature and are observed to exhibit excellent fluorescence properties.

\section{Experimental Section}

General Procedures. All the chemicals used were that of analytical grade. Melting points were uncorrected, determined in open capillary. Purity of the compounds was checked by TLC on silica gel and compounds were purified by using column chromatography. ${ }^{1} \mathrm{H}$ NMR spectra was recorded on a Bruker supercon FT NMR (400 MHz) spectrometer in $\mathrm{CDCl}_{3}$ or DMSO- $d_{6}$ and, TMS as an internal standard. The chemical shifts are expressed in $\delta$ units. IR spectra was recorded by using JASCO FT/IR-300 E spectrometer from a KBr pelleted sample. Mass spectras was recorded on a JEOL SX 102/DA-6000 (10 kV) FAB mass spectrometer. Fluorescence spectra was recorded by F-7000 FL (SL. NO 1911-004, ROM 5J14000 03) Spectrophoto meter. The Elemental analysis was obtained by "Elementar vario EL-III instrument".

Synthesis of ethyl benzocoumarin-3- carboxylate (2). A mixture of 2-hydroxy-1naphthaldehyde $(2.9 \mathrm{mmol}) \mathbf{1}$, an equivalent amount of diethyl malonate $(2.9 \mathrm{mmol})$, and catalytic amount of piperidine in ethanol $(30 \mathrm{~mL})$ was refluxed for 30 minutes on water bath ${ }^{14}$. After the reaction was complete, the reaction mixture was cooled to room temperature and poured into crushed ice with stirring. The precipitate obtained was then filtered, washed with water, dried and recrystallised using ethanol to get pure 2 .

Ethyl benzocoumarin-3- carboxylate (2). White Yellow Crystalline Solid. Yield 90\%. m.p. $115-116^{\circ} \mathrm{C}\left(117-118^{\circ} \mathrm{C}\right.$ Reported. Ref-14 \& 16) (ethanol); ${ }^{1} \mathrm{H}$ NMR $\left(400 \mathrm{MHz}, \mathrm{CDCl}_{3}\right) \delta$ (ppm): 1.4 (s, 3H, $\left.\mathrm{CH}_{3}\right), 4.4$ (q, 2H, $\left.\mathrm{H}_{2}\right), 7.4$ (m, 6H, ArH ), $9.2(\mathrm{~s}, 1 \mathrm{H}, \mathrm{CH}):{ }^{13} \mathrm{C}$ NMR $(300$ $\left.\mathrm{MHz}, \mathrm{CDCl}_{3}\right) \delta(\mathrm{ppm}): 163(\mathrm{C}=\mathrm{O}$ ester $), 160(\mathrm{C}=\mathrm{O}$ pyrone $), 150.4,149.7,148.7,130.6,126.6$, 124.2, 124.0, 121.2, 121, 120, 114.5, 115, 58.8, 26: IR (KBr) $v\left(\mathrm{~cm}^{-1}\right): 1765$ (s) (C=O), 1750 (s) $(\mathrm{C}=\mathrm{O}$ ester): $\mathrm{MS}(\mathrm{m} / \mathrm{z}): 269(\mathrm{M}+1)$.

Synthesis of benzocoumarin-3-carbohydrazide (3). A mixture of ethyl benzocoumarin-3carboxylate $2(3.8 \mathrm{mmol})$ and hydrazine hydrate $(3.8 \mathrm{mmol})$ was dissolved in ethanol and refluxed on waterbath for $2 \mathrm{hr}$. After the reaction was complete, the reaction mixture was cooled to room temperature and poured into crushed ice with stirring. The separated solid was filtered, washed with water, dried and recrystallised with ethanol to get pure yellow compound 3 .

Benzocoumarin-3-carbohydrazide (3). Yellow Crystalline Solid. Yield 95\%; m.p.260-262 ${ }^{\circ} \mathrm{C}$ (chloroform); ${ }^{1} \mathrm{H}$ NMR (400 MHz, DMSO- $\left.d_{6}\right) \delta(\mathrm{ppm}): 4.7\left(\mathrm{~s}, 2 \mathrm{H}, \mathrm{NH}_{2}\right.$ ), 7.7 (t, 2H, $J=7.9 \mathrm{~Hz}$ ), $7.8(\mathrm{~m}, 1 \mathrm{H}, J=1.2 \mathrm{~Hz}), 8.1(\mathrm{~d}, 1 \mathrm{H}, J=8.1 \mathrm{~Hz}), 8.3(\mathrm{~d}, 1 \mathrm{H}, J=9.1 \mathrm{~Hz}), 8.63(\mathrm{~d}, 1 \mathrm{H}, J=8.5 \mathrm{~Hz}$

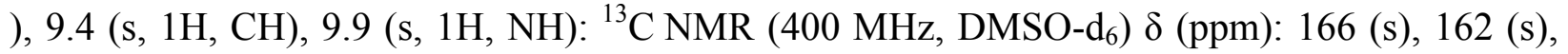
152 (d), 150.5 (s), 145 (d), 136 (d), 130.8 (s) 130 (d), 129.8 (d), 129.8 (s), 127.8 (d), 117 (d), 114.5 (s), 112 (s) (: IR (KBr) v ( $\left.\mathrm{cm}^{-1}\right)$ : $1765(\mathrm{~s})\left(\mathrm{C}=\mathrm{O}\right.$ pyrone), $3020(\mathrm{~m})\left(\mathrm{NH}_{2}\right), 1605(\mathrm{~s})(\mathrm{C}=\mathrm{O})$ : 
MS (m/z): $255(\mathrm{M}+1)$. Anal. Calcd. For $\mathrm{C}_{14} \mathrm{H}_{10} \mathrm{~N}_{2} \mathrm{O}_{3}$ : C, 66.14; H, 3.96; N 11.02. Found: C, 66.03; H, 3.83; N10.86.

Synthesis of 3-Benzocoumarin- 5- (undecanyl) - 1, 3, 4 oxadiazole (4a). To the mixture of benzocoumarin-3-carbohydrazide $3(2.0 \mathrm{mmol})$, lauric acid $(2.0 \mathrm{mmol}) 10 \mathrm{ml}$ of phosphorous oxychloride was added and then it was refluxed for about 12-15 hr on water bath and cooled to room temperature. The mixture was poured into crushed ice with stirring and it was neutralized by using saturated sodium bicarbonate solution. The yellow precipitate obtained was filtered, washed with water, dried and purified through column chromatography by using ethyl acetate and petroleum ether (1:9) as the elutent. Similarly the compounds $\mathbf{4 b - e}$ were synthesized.

3-Benzocoumarin- 5- (undecanyl) - 1, 3, 4 oxadiazole (4a). Yellow Solid. Yield 60\%; m.p.71$73{ }^{\circ} \mathrm{C}$ (ethyl acetate); ${ }^{1} \mathrm{H}$ NMR $\left(300 \mathrm{MHz}, \mathrm{DMSO}-d_{6}\right) \delta(\mathrm{ppm}): 9.44(\mathrm{~s}, 1 \mathrm{H}, \mathrm{CH}), 8.68(\mathrm{~d}, 1 \mathrm{H}, J=$ $8.2 \mathrm{~Hz}), 8.33(\mathrm{~d}, 1 \mathrm{H}, J=9.2 \mathrm{~Hz}), 8.10(\mathrm{~d}, 1 \mathrm{H}, J=7.4 \mathrm{~Hz}), 7.77(\mathrm{~d}, 1 \mathrm{H}, J=7.2 \mathrm{H}), 7.68(\mathrm{t}, 2 \mathrm{H}, J$ $=8.5 \mathrm{~Hz}), 2.95(\mathrm{t}, 2 \mathrm{H}, J=7.1 \mathrm{~Hz}), 1.7\left(\mathrm{~s}, 2 \mathrm{H}, \mathrm{CH}_{2}\right), 1.2(\mathrm{~s}, 16 \mathrm{H},-\mathrm{CH} 2-$ protons $), 0.81(\mathrm{~s}, 3 \mathrm{H}$, $\mathrm{CH}_{3}$ ): ${ }^{13} \mathrm{CNMR}\left(400 \mathrm{MHz}, \mathrm{DMSO}-d_{6}\right) \delta(\mathrm{ppm}): 167.8$ (s), 160.6 (s), 156.0 (s), 154.6 (s), 141.3 (d), 136.0 (d), 130.4 (s), 129.4 (d), 129.4 (d), 129.3 (s), 126.8 (d), 122.1 (d), 116.6 (d), 112.6 (s), $111.1(\mathrm{~s}), 31.2(\mathrm{t}), 29.4(\mathrm{t}), 29.4(\mathrm{t}), 29.3(\mathrm{t}), 29.3(\mathrm{t}), 28.6(\mathrm{t}), 28.5(\mathrm{t}), 26.1(\mathrm{t}), 24.8(\mathrm{t}), 22.5(\mathrm{t})$, 14.1 (q): IR (KBr) v ( $\left.\mathrm{cm}^{-1} 1\right): 1765(\mathrm{~s})(\mathrm{C}=\mathrm{O}$ pyrone), $1592(\mathrm{~s})(\mathrm{C}=\mathrm{N}) ; \mathrm{MS}(\mathrm{m} / \mathrm{z}): 419(\mathrm{M}+1)$. Anal. Calcd. For $\mathrm{C}_{26} \mathrm{H}_{30} \mathrm{~N}_{2} \mathrm{O}_{3}$ : C, 74.61; H, 7.22; N, 6.69. Found: C, 74.67; H, 7.04; N 6.66.

3-Benzocoumarin- 5- (tridecanyl)-1, 3, 4-oxadiazole (4b). Yellow Solid. Yield 50\%; m.p.86$88{ }^{\circ} \mathrm{C}$ (ethyl acetate); ${ }^{1} \mathrm{H}$ NMR $\left(400 \mathrm{MHz}, \mathrm{CDCl}_{3}\right) \delta(\mathrm{ppm}): 9.41$ (s, $\left.1 \mathrm{H}, \mathrm{CH}\right), 8.4(\mathrm{~d}, 1 \mathrm{H}, J=8.5$ $\mathrm{Hz}), 8.14(\mathrm{~d}, 1 \mathrm{H}, J=9.0 \mathrm{~Hz}), 7.98(\mathrm{~d}, 1 \mathrm{H}, J=8.1 \mathrm{~Hz}), 7.80(\mathrm{t}, 1 \mathrm{H}, J=7.2 \mathrm{~Hz}), 7.67(\mathrm{t}, 1 \mathrm{H}, J=$ $7.5 \mathrm{~Hz}), 7.55(\mathrm{~d}, 1 \mathrm{H}, J=9.0 \mathrm{~Hz}), 3.02(\mathrm{t}, 2 \mathrm{H}, J=7.7 \mathrm{~Hz}), 1.92(\mathrm{~m}, 2 \mathrm{H}, J=7.4 \mathrm{~Hz}), 1.46(\mathrm{t}, 2 \mathrm{H}$, $J=7.7 \mathrm{~Hz}), 1.2\left(\mathrm{~s}, 18 \mathrm{H},-\mathrm{CH}_{2}-\right.$ protons), 0.90 (t, 3H, $\left.J=6.3 \mathrm{~Hz}\right):{ }^{13} \mathrm{CNMR}\left(400 \mathrm{MHz}, \mathrm{DMSO}-d_{6}\right)$ $\delta(\mathrm{ppm}): 167.8$ (s), 161 (s), 156.4 (s), 154.9 (s), 141.5 (d), 136 (d), 130.4 (s), 129.5 (d), 129.4 (d), $129.3(\mathrm{~s}), 127(\mathrm{~d}), 122(\mathrm{~d}), 117(\mathrm{~d}), 112.8(\mathrm{~s}), 111.4(\mathrm{~s}), 31.7(\mathrm{t}), 29.5(\mathrm{t}), 29.4(\mathrm{t}), 29.4(\mathrm{t}), 29.4$ $(\mathrm{t}), 29.3(\mathrm{t}), 29.1(\mathrm{t}), 28.9(\mathrm{t}), 28.7(\mathrm{t}), 26.3(\mathrm{t}), 25(\mathrm{t}), 22.5(\mathrm{t}), 14.4(\mathrm{q}): \operatorname{IR}(\mathrm{KBr}) v\left(\mathrm{~cm}^{-1} 1\right): 1765$ (s) $\left(\mathrm{C}=\mathrm{O}\right.$ pyrone), $1602(\mathrm{~s})(\mathrm{C}=\mathrm{N}) ; \mathrm{MS}(\mathrm{m} / \mathrm{z})$ : $447.5(\mathrm{M}+1)$. Anal. Calcd. For $\mathrm{C}_{28} \mathrm{H}_{34} \mathrm{~N}_{2} \mathrm{O}_{3}$ : $\mathrm{C}$, 75.31; H, 7.67; N, 6.27. Found: C, 75.19; H, 7.56; N 6.40.

3-Benzocoumarin-5- (pentadecanyl) - 1, 3, 4-oxadiazole (4c). Yellow Solid. Yield 60\%; m.p.90-92 ${ }^{\circ} \mathrm{C}$ (ethyl acetate); ${ }^{1} \mathrm{H}$ NMR (400 MHz, DMSO- $\left.d_{6}\right) \delta(\mathrm{ppm}): 9.49(\mathrm{~s}, 1 \mathrm{H}, \mathrm{CH}), 8.71$ (d, $1 \mathrm{H}, J=8.5 \mathrm{~Hz}), 8.35(\mathrm{~d}, 1 \mathrm{H}, J=8.9 \mathrm{~Hz}), 8.12(\mathrm{~d}, 1 \mathrm{H}, J=8.1 \mathrm{~Hz}), 7.82(\mathrm{~d}, 1 \mathrm{H}, J=7.7 \mathrm{~Hz}), 7.70$ $(\mathrm{m}, 2 \mathrm{H}, J=8.3 \mathrm{~Hz}), 2.90\left(2 \mathrm{H}, \mathrm{t}, \mathrm{CH}_{2}\right), 1.22(\mathrm{~d}, 26 \mathrm{H}, J=10.2 \mathrm{~Hz}), 0.84(\mathrm{t}, 3 \mathrm{H}, J=6.0 \mathrm{~Hz}):{ }^{13} \mathrm{C}$ NMR (400 MHz, DMSO- $d_{6}$ ) $\delta$ (ppm): 167.8 (s), $161.1(\mathrm{~s}), 156.8$ (s), 155.2 (s), 141.5 (d), 136 (d), 130.5 (s), 129.5 (d), 129.4 (d), 129.4 (s), 127.4 (d), 122.1 (d), 117.3 (d), 112.8 (s), 114. (s), 31 (t), $29.5(\mathrm{t}), 29.5(\mathrm{t}), 29.5(\mathrm{t}), 29.4(\mathrm{t}), 29.4(\mathrm{t}), 29.3(\mathrm{t}), 29.1(\mathrm{t}), 29(\mathrm{t}), 29(\mathrm{t}), 28.8(\mathrm{t}), 26.6(\mathrm{t}), 25.4$ $(\mathrm{t}), 22.5(\mathrm{t}), 14.4(\mathrm{q}): \operatorname{IR}(\mathrm{KBr}) \vee\left(\mathrm{cm}^{-1}\right)$ : $1765(\mathrm{~s})(\mathrm{C}=\mathrm{O}$ pyrone $), 1612(\mathrm{~s})(\mathrm{C}=\mathrm{N}) ; \mathrm{MS}(\mathrm{m} / \mathrm{z})$ : 475.6 (M+1). Anal. Calcd. For $\mathrm{C}_{30} \mathrm{H}_{38} \mathrm{~N}_{2} \mathrm{O}_{3}$ : C, 75.92; H, 8.07; N, 5.9. Found: C, 75.83; H, 7.94; N 6.02.

3-Benzocoumarin- 5- (heptadecanyl) - 1, 3, 4-oxadiazole (4d). Yellow Solid. Yield 50\%; m.p. $75-77^{\circ} \mathrm{C}$ (ethyl acetate); ${ }^{1} \mathrm{H}$ NMR $\left(300 \mathrm{MHz}, \mathrm{CDCl}_{3}\right) \delta(\mathrm{ppm}): 9.4(\mathrm{~s}, 1 \mathrm{H}, \mathrm{CH}), 8.39(\mathrm{~d}, 1 \mathrm{H}$, 
$J=8.4 \mathrm{~Hz}), 8.14(\mathrm{~d}, 1 \mathrm{H}, J=9.0 \mathrm{~Hz}), 7.97(\mathrm{~d}, 1 \mathrm{H}, J=8.9 \mathrm{~Hz}), 7.80(\mathrm{t}, 1 \mathrm{H}, J=7.3 \mathrm{~Hz}), 7.66(\mathrm{t}$, $1 \mathrm{H}, J=7.4 \mathrm{~Hz}), 7.54(\mathrm{~d}, 1 \mathrm{H}, J=9.0 \mathrm{~Hz}), 3.02(\mathrm{t}, 2 \mathrm{H}, J=7.6 \mathrm{~Hz}), 2.37$ (t, 2H, $J=7.5 \mathrm{~Hz}), 1.92$ $(\mathrm{t}, 2 \mathrm{H}, J=6.8 \mathrm{~Hz}), 1.4\left(\mathrm{~s}, 2 \mathrm{H}, \mathrm{CH}_{2}\right), 1.2\left(\mathrm{~s}, 24 \mathrm{H}, \mathrm{CH}_{2}\right.$ protons), $0.88(\mathrm{t}, 3 \mathrm{H}, J=6.5 \mathrm{~Hz})$; ${ }^{13} \mathrm{CNMR}\left(400 \mathrm{M} \mathrm{Hz}, \mathrm{DMSO}-d_{6}\right) \delta$ (ppm): 167.8 (s), 161 (s), 156.9 (s), 155.5 (s), 141.5 (d), 136.2 (d), 130.5 (s), 129.6 (d), 129.4 (d), 129.4 (s), 127.5 (d), 122.1 (d), 117.3 (d), 112.8 (s), 111.8 (s), $31.7(\mathrm{t}), 29.8(\mathrm{t}), 29.5(\mathrm{t}), 29.4(\mathrm{t}), 29.4(\mathrm{t}), 29.4(\mathrm{t}), 29.4(\mathrm{t}), 29.4(\mathrm{t}), 29.3(\mathrm{t}), 29.1(\mathrm{t}), 28.9(\mathrm{t})$, $28.7(\mathrm{t}), 28.6(\mathrm{t}), 26.6(\mathrm{t}), 25.3(\mathrm{t}), 22.9(\mathrm{t}), 14.6(\mathrm{q}): \operatorname{IR}(\mathrm{KBr}) \vee\left(\mathrm{cm}^{-1}\right): 1765(\mathrm{~s})(\mathrm{C}=\mathrm{O}$ pyrone $)$, $1602(\mathrm{~s})(\mathrm{C}=\mathrm{N})$; MS (m/z): $503(\mathrm{M}+1)$. Anal. Calcd. For $\mathrm{C}_{32} \mathrm{H}_{42} \mathrm{~N}_{2} \mathrm{O}_{3}: \mathrm{C}, 76.46 ; \mathrm{H}, 8.42 ; \mathrm{N}, 5.57$. Found: C,76.35; H, 8.38; N 5.36.

3-Benzocoumarin-5-( nona- 9,10-decenyl) -1, 3, 4-oxadiazole (4e). Yellow Semi Solid. Yield $50 \%$ (ethyl acetate); ${ }^{1} \mathrm{H} \mathrm{NMR}\left(400 \mathrm{MHz}, \mathrm{CDCl}_{3}\right) \delta(\mathrm{ppm}): 9.41$ (s, $\left.1 \mathrm{H}, \mathrm{CH}\right), 8.40(\mathrm{~d}, 1 \mathrm{H}, J=8.4$ $\mathrm{Hz}), 8.14(\mathrm{~d}, 1 \mathrm{H}, J=9.0 \mathrm{~Hz}), 7.98(\mathrm{~d}, 2 \mathrm{H}, J=8.0 \mathrm{~Hz}), 7.80(\mathrm{t}, 2 \mathrm{H}, J=7.2 \mathrm{~Hz}), 7.60(\mathrm{t}, 1 \mathrm{H}, J=$ $7.5 \mathrm{~Hz}), 7.54(\mathrm{~d}, 1 \mathrm{H}, J=9.0 \mathrm{~Hz}), 7.14(\mathrm{~m}, 1 \mathrm{H}, J=6.1 \mathrm{~Hz}), 5.38\left(\mathrm{~m}, 2 \mathrm{H}, \mathrm{CH}_{2}\right), 3.02(\mathrm{t}, 2 \mathrm{H}, J=$ 7.8), 1.97 (s, 4H, $\left.\mathrm{CH}_{2}\right), 1.92(\mathrm{t}, 2 \mathrm{H}, J=7.4 \mathrm{~Hz}), 1.46(\mathrm{~m}, 4 \mathrm{H}, J=6.5 \mathrm{~Hz}),, 1.34\left(\mathrm{~m}, 6 \mathrm{H}, \mathrm{CH}_{2}-\right.$ protons), $1.29(\mathrm{~m}, 10 \mathrm{H}, J=12.4 \mathrm{~Hz}), 0.88(\mathrm{t}, 3 \mathrm{H}, J=6.5 \mathrm{~Hz}):,{ }^{13} \mathrm{C}$ NMR (400 MHz, DMSO- $\left.d_{6}\right)$ $\delta(\mathrm{ppm}): 167.4$ (s), 161 (s), 156.4 (s), 155.3 (s), 141.2 (d), 136.2 (d), 130.5 (s), 129.6 (d), 129.4 (d), 129.4 (s), 127.5 (d), 127.1 (d), 117.3 (d), 112.8 (s), 111.8 (s), 36.6 (d), 36.6 (d), 31.9 (d), $29.8(\mathrm{t}), 29.5(\mathrm{t}), 29.4(\mathrm{t}), 29.4(\mathrm{t}), 29.4(\mathrm{t}), 29.3(\mathrm{t}), 29.1(\mathrm{t}), 28.9(\mathrm{t}), 28.8(\mathrm{t}), 28.6(\mathrm{t}), 26.7(\mathrm{t})$, $25.3(\mathrm{t}), 22.9(\mathrm{t}), 14.6(\mathrm{q})$ : IR (KBr) $v\left(\mathrm{~cm}^{-1}\right.$; $1765(\mathrm{~s})(\mathrm{C}=\mathrm{O}$ pyrone $), 1624(\mathrm{~s})(\mathrm{C}=\mathrm{N}) ; \mathrm{MS}(\mathrm{m} / \mathrm{z})$ : $501.13(\mathrm{M}+1)$. Anal. Calcd. For $\mathrm{C}_{32} \mathrm{H}_{40} \mathrm{~N}_{2} \mathrm{O}_{3}$ : C, 76.77; H, 8.05; N, 5.6. Found: C, 76.59; H, 7.76 ; N 5.46 .

\section{Acknowledgements}

We are thankful to Department of Post Graduate Studies and Research in Chemistry, Kuvempu University, Shankaraghatta, Karnataka 577451, India for providing laboratory facilities and Karnataka University, Dharwad, Karnataka, India for fluorescence spectral data. We are also thankful to Dept of Chemistry University of Mysore, Karnataka-India-57006 for providing elemental analytical data. We are thankful to Indian Institute of Science, Bangalore, India for Spectral data. Finally we are thankful to Vinay K.M Lecturer Dept of English for proofreading.

\section{References}

1. Hunger, K.; Industrial dyes. Weiheim, Germany: Wiley-VCH, 2003; pp 569-577.

2. Berlman, I. B.; Handbook of fluorescence spectra of aromatic molecules. New York: Academic Press, 1971.

3. (a) Kodiro, K.; Inoue, Y. A. J. Am Chem. Soc. 2003, 125, 421. (b) Yamaguchi, S.; Akiyama, S.; Tamao, K. J. Am. Chem. Soc. 2000, 122, 6793. 
4. Harvey, M. D.; Bablekis, V.; Banks, P. R.; Skinner, C. D. J Chromatogr B. 2001, 754, 345.

5. Casley-Smith, J. R. J .B. Lippincott Company: Sydney. 1986, 181.

6. Xiao, S.; Yi, T.; Li, F.; Huang, C. Tetrahedron Lett. 2005, 46, 9009.

7. Saleh, M.A.; Kamel, A.; El-Demerdash, A.; Jones, J. Chemosphere 1998, 36, 1543.

8. Yu, H.; Mizufune, H.; Uenaka, K.; Moritoki, T.; Koshima, H. Tetrahedron 2005, 61, 8932.

9. Drexhage, K. H.; Topics in Applied Physics; Springer-Verlag: New York, 1973, Vol. 1.

10. Jones, II, G.; Jackson, W. R.; Choi, C.; Bergmark, W. R. J. Phys. Chem. 1985, 89, 294.

11. Gikas, E.; Parissi-Poulou, M.; Kazanis, M.; Vavagianis, A. Anal. Chim. Acta. 2003, 489, 153.

12. Ammar, H.; Fery-Forgues, S.; El, Gharbi, R. Dyes Pigments 2003, 57, 259.

13. Sastry, S. Biophys. Chem. 2001, 91, 191.

14. Chiyomi, M..; Toshinobu, M..; Yasuko, K..; Kenichiro, T..; Hideyuki, Y..; Hitoshi, N.; Masatoshi, Y..; and Akira, T.. Chem. Pharm. Bull. 2005, 53, 750.

15. Suresha Kumara, T. H.; Srinivasa, A.; Mahadevan, K. M.; Basavaraj Padmashali Indian Journal of Heterocyclic Chemistry 2007, 17, 117.

16. Bogdal, D. J. Chem. Research (S). 1998, 468. 